\title{
A note on chaos for flow
}

\author{
Zhongxuan Yang \\ Nanchang Institute of Science and Technology, NanChang 330031, China \\ email: yangjingmike@163.com
}

Keywords: quasi-weakly almost periodic points; ergodicly sensitive ; $C^{0}$-flow; measure center

Abstract.In this paper,we consider a continuous flow $\varphi: R \times X \rightarrow X$, where $X$ is a compact metric space,and we prove that $\varphi$ is Li-Yorke chaotic if and only if $\varphi \times \varphi$ is Li-Yorke chaotic; $\varphi$ is distributional chaotic if and only if $\varphi \times \varphi$ is distributional chaotic.

\section{Introduction}

In 1975,Li and Yorke first gave the definition of chaos(see [3]),the definition opened the door on researching chaos,many scholars began to explore the chaos and give the different notions and concepts of chaos.In 1994,Schweizer and Smital defined a new chaos named distributional chaos(see[4,6]).The scholar's effort is to clarify the essence of the complexity of dynamical systems. Nowadays to investigate the chaotic behavior of dynamical systems has become a hot subject.

\section{Preliminaries}

Let $(X, d)$ be a compact metric space with metric $d$, write $R=(-\infty,+\infty)$. We call $\varphi: R \times X \rightarrow X$ is a continuous flow if $\varphi$ satisfies the following conditions;

(1) $\varphi(0, x)=x, \forall x \in X$;(2) $\varphi(t, \cdot): X \rightarrow X, \forall t \in R$ is homeomorphism.

(3) $\varphi(t, \varphi(s, x))=\varphi(s+t, x)), \forall s, t \in R$.

The product metric $\rho$ on the product space $X \times X$ is defined by $\rho\left((x, y),\left(x^{\prime}, y^{\prime}\right)\right)=\max \left\{d\left(x, x^{\prime}\right), d\left(y, y^{\prime}\right)\right\}$ for any $(x, y),\left(x^{\prime}, y^{\prime}\right) \in X \times X$.

A continuous flow $\varphi \times \varphi: R \times X \times X \rightarrow X \times X$ is defined by $\varphi \times \varphi(t,(x, y))=(\varphi(t, x), \varphi(t, y)), \forall x \in X$ for any $t \in R$ and $(x, y) \in X \times X$.

$\varphi$ is said to be Li-Yorke chaotic if there exists an uncountable set $D \subset X$ such that for any pair $(x, y) \in D \times D$ with $x \neq y$,

(1) $\liminf _{t \rightarrow \infty} d(\varphi(t, x), \varphi(t, y))=0$; (2) $\limsup _{t \rightarrow \infty} d(\varphi(t, x), \varphi(t, y))>0$

For any real number $s>0, x, y \in X$, let

(1) $\underline{F}_{x y}(s)=\liminf _{t \rightarrow \infty} \frac{1}{t} \int_{0}^{t} \chi_{[0, s]}(d(\varphi(t, x), \varphi(t, y))) d t$

(2) $\bar{F}_{x y}(s)=\limsup _{t \rightarrow \infty} \frac{1}{t} \int_{0}^{t} \chi_{[0, s]}(d(\varphi(t, x), \varphi(t, y))) d t$

Where $\chi_{A}(x)$ is 1 if $x \in A$, and $\chi_{A}(x)$ is 0 if $x \notin A$.Obviously $\underline{F}_{x y}(s)$ and $\bar{F}_{x y}(s)$ are both nondecreasing functions. We call $(x, y) \in X \times X$ is a pair displaying distributional chaos if (1) $\underline{F}_{x y}(\alpha)=0$,for some $\alpha>0 ;(2) \bar{F}_{x y}(s)=1$, for any $s>0$.

$\varphi$ is said to display distributional chaotic if there exists an uncountable set $D \subset X$ such that any two different points in $D$ is a pair displaying distributional chaos.For simplicity,let $\varepsilon_{t}(\varphi, x, y, s)=\int_{0}^{t} \chi_{[0, s]}(d(\varphi(t, x), \varphi(t, y))) d t$, 
$\underline{F}(\varphi, x, y, s)=\liminf _{t \rightarrow \infty} \frac{1}{t} \varepsilon_{t}(\varphi, x, y, s), \bar{F}(\varphi, x, y, s)=\limsup _{t \rightarrow \infty} \frac{1}{t} \varepsilon_{t}(\varphi, x, y, s)$

\section{Results}

Theorem 3.3 Let $(X, d)$ be a compact metric space , $\varphi: R \times X \rightarrow X$ be a continuous flow.Then $\varphi$ is Li-Yorke chaotic if and only if $\varphi \times \varphi$ is Li-Yorke chaotic.

Proof Suppose $\varphi$ is Li-Yorke chaotic.Then there exists an uncountable set $D \subset X$ such that for any pair $(x, y) \in D \times D$ with $x \neq y$,

$\liminf _{t \rightarrow \infty} d(\varphi(t, x), \varphi(t, y))=0$ and $\quad \limsup _{t \rightarrow \infty} d(\varphi(t, x), \varphi(t, y))>0$.

Let $D^{\prime}=D \times D$, then $D^{\prime} \subset X \times X \quad$ is an uncountable set.Taking $\mu=(x, y)$, $v=\left(x^{\prime}, y^{\prime}\right) \in D^{\prime}$, and $\mu \neq v$. Noting that $x, y, x^{\prime}, y^{\prime} \in D, x \neq x^{\prime}$,or $y \neq y^{\prime}$,

then we have if $x \neq x^{\prime}$, and $y \neq y^{\prime}$,

$\liminf _{t \rightarrow \infty} d\left(\varphi(t, x), \varphi\left(t, x^{\prime}\right)\right)=0$ and $\quad \limsup _{t \rightarrow \infty} d\left(\varphi(t, x), \varphi\left(t, x^{\prime}\right)\right)=0$.

$\liminf _{t \rightarrow \infty} d\left(\varphi(t, y), \varphi\left(t, y^{\prime}\right)\right)=0$ and $\quad \limsup _{t \rightarrow \infty} d\left(\varphi(t, y), \varphi\left(t, y^{\prime}\right)\right)>0$.

If $x=x^{\prime}$, and $y \neq y^{\prime}$,

$\liminf _{t \rightarrow \infty} d\left(\varphi(t, x), \varphi\left(t, x^{\prime}\right)\right)=0$ and $\quad \limsup _{t \rightarrow \infty} d\left(\varphi(t, x), \varphi\left(t, x^{\prime}\right)\right)=0$.

$\liminf _{t \rightarrow \infty} d\left(\varphi(t, y), \varphi\left(t, y^{\prime}\right)\right)=0$ and $\quad \limsup _{t \rightarrow \infty} d\left(\varphi(t, y), \varphi\left(t, y^{\prime}\right)\right)>0$.

If $x \neq x^{\prime}$, and $y=y^{\prime}$,

$$
\begin{aligned}
& \liminf _{t \rightarrow \infty} d\left(\varphi(t, x), \varphi\left(t, x^{\prime}\right)\right)=0 \text { and } \quad \limsup _{t \rightarrow \infty} d\left(\varphi(t, x), \varphi\left(t, x^{\prime}\right)\right)>0 . \\
& \liminf _{t \rightarrow \infty} d\left(\varphi(t, y), \varphi\left(t, y^{\prime}\right)\right)=0 \text { and } \quad \limsup _{t \rightarrow \infty} d\left(\varphi(t, y), \varphi\left(t, y^{\prime}\right)\right)=0 \text {. }
\end{aligned}
$$

Hence

$$
\begin{gathered}
\liminf _{t \rightarrow \infty} \rho(\varphi \times \varphi(t, \mu), \varphi \times \varphi(t, v)) \\
=\liminf _{t \rightarrow \infty} \max \left\{d\left(\varphi(t, x), \varphi\left(t, x^{\prime}\right)\right), d\left(\varphi(t, y), \varphi\left(t, y^{\prime}\right)\right)\right\}=0 \\
\limsup _{t \rightarrow \infty} \rho(\varphi \times \varphi(t, \mu), \varphi \times \varphi(t, v)) \\
=\limsup _{t \rightarrow \infty} \max \left\{d\left(\varphi(t, x), \varphi\left(t, x^{\prime}\right)\right), d\left(\varphi(t, y), \varphi\left(t, y^{\prime}\right)\right)\right\}=0
\end{gathered}
$$

Consequently, $\varphi \times \varphi$ is Li-Yorke chaotic.

Assume $\varphi \times \varphi$ is Li-Yorke chaotic.Then there exists an uncountable set $D \subset X \times X$ such that for any pair $(\mu, v) \in D \times D$ with $\mu \neq v$, then we have

$\liminf _{t \rightarrow \infty} \rho(\varphi \times \varphi(t, \mu), \varphi \times \varphi(t, v))=0 \quad \limsup _{t \rightarrow \infty} \rho(\varphi \times \varphi(t, \mu), \varphi \times \varphi(t, v))>0$

We define the map $\pi_{1}: D \rightarrow X$ as $\pi_{1}(x, y)=x, \pi_{2}: D \rightarrow Y$ as $\pi_{2}(x, y)=y$,

for any $(x, y) \in D$.As $D$ is an uncountable set, $\pi_{1}(D)$ or $\pi_{2}(D)$ is an uncountable set.Generally,we suppose $\pi_{1}(D)$ to be an uncountable set. Let $x, x^{\prime} \in \pi_{1}(D)$, and $x \neq x^{\prime}$, then exists $y \in \pi_{2}(D)$, such that $(x, y),\left(x^{\prime}, y^{\prime}\right) \in D$. Hence we have

$$
\begin{aligned}
& \liminf _{t \rightarrow \infty} \rho\left(\varphi \times \varphi(t,(x, y)), \varphi \times \varphi\left(t,\left(x^{\prime}, y^{\prime}\right)\right)\right)=\liminf _{t \rightarrow \infty} d\left(\varphi(t, x), \varphi\left(t, x^{\prime}\right)\right)=0 \\
& \limsup _{t \rightarrow \infty} \rho\left(\varphi \times \varphi(t,(x, y)), \varphi \times \varphi\left(t,\left(x^{\prime}, y^{\prime}\right)\right)\right)=\limsup _{t \rightarrow \infty} d\left(\varphi(t, x), \varphi\left(t, x^{\prime}\right)\right)>0
\end{aligned}
$$


Therefore $\varphi$ is Li-Yorke chaotic.This completes the proof.

Theorem 3.4 Let $(X, d)$ be a compact metric space, $\varphi: R \times X \rightarrow X$ be a continuous flow.Then $\varphi$ is distributional chaotic if and only if $\varphi \times \varphi$ is distributional chaotic.

Proof Assume $\varphi$ is distributional chaotic. Then there exists an uncountable set $D \subset X$ such that for any pair $(x, y) \in D \times D$ with $x \neq y$,

$\underline{F}(\varphi, x, y, p)=0$, for some $p>0 ; \bar{F}(\varphi, x, y, s)=1$, for any $s>0$.

Let $D^{\prime}=D \times D$, then $D^{\prime} \subset X \times X$ is an uncountable set. Let $\mu=(x, y)$, $v=\left(x^{\prime}, y^{\prime}\right) \in D^{\prime}$, and $\mu \neq v$. Noting that $x, y, x^{\prime}, y^{\prime} \in D, x \neq x^{\prime}$,or $y \neq y^{\prime}$,

then we have if $x \neq x^{\prime}$, and $y \neq y^{\prime}$,

$\underline{F}\left(\varphi, x, x^{\prime}, p_{1}\right)=0$, for some $p_{1}>0 ; \bar{F}\left(\varphi, x, x^{\prime}, s\right)=1$, for any $s>0$.

$\underline{F}\left(\varphi, y, y^{\prime}, p_{2}\right)=0$, for some $p_{2}>0 ; \bar{F}\left(\varphi, y, y^{\prime}, s\right)=1$, for any $s>0$.

If $x \neq x^{\prime}$, and $y=y^{\prime}$,

$$
\begin{array}{cl}
\underline{F}\left(\varphi, x, x^{\prime}, p_{1}\right)=0, \text { for some } p_{1}>0 ; \bar{F}\left(\varphi, x, x^{\prime}, s\right)=1 \text {, for any } s>0 . \\
\underline{F}\left(\varphi, y, y^{\prime}, p_{2}\right)=0, \text { for some } p_{2}>0 ; \bar{F}\left(\varphi, y, y^{\prime}, s\right)=0 \text {, for any } s>0 .
\end{array}
$$

If $x=x^{\prime}$, and $y \neq y^{\prime}$,

$\underline{F}\left(\varphi, x, x^{\prime}, p_{1}\right)=0$, for some $p_{1}>0 ; \bar{F}\left(\varphi, x, x^{\prime}, s\right)=0$, for any $s>0$.

$\underline{F}\left(\varphi, y, y^{\prime}, p_{2}\right)=0$, for some $p_{2}>0 ; \bar{F}\left(\varphi, y, y^{\prime}, s\right)=1$, for any $s>0$.

Let $p=\max \left\{p_{1}, p_{2}\right\}$, then

and further

$$
\varepsilon_{t}(\varphi \times \varphi, \mu, v, p) \leq \varepsilon_{t}\left(\varphi, x, x^{\prime}, p_{1}\right)+\varepsilon_{t}\left(\varphi, y, y^{\prime}, p_{2}\right)
$$

$$
\underline{F}(\varphi \times \varphi, \mu, v, p)=\underline{F}\left(\varphi, x, x^{\prime}, p_{1}\right)+\underline{F}\left(\varphi, y, y^{\prime}, p_{2}\right)=0+0=0
$$

Obviously, $\bar{F}(\varphi \times \varphi, \mu, \nu, s)=1$, for any $s>0$.

Therefore $\varphi \times \varphi$ is distributional chaotic.

Suppose $\varphi \times \varphi$ is distributional chaotic. Then there exists an uncountable set $D \subset X \times X$ such that for any pair $(\mu, v) \in D \times D$ with $\mu \neq v$,

$\underline{F}(\varphi \times \varphi, \mu, \nu, p)=0$, for some $p>0 ; \bar{F}(\varphi \times \varphi, \mu, \nu, s)=1$, for any $s>0$.

We define the map $\pi_{1}: D \rightarrow X$ as $\pi_{1}(x, y)=x, \pi_{2}: D \rightarrow Y$ as $\pi_{2}(x, y)=y$,

for any $(x, y) \in D$. As $D$ is an uncountable set, $\pi_{1}(D)$ or $\pi_{2}(D)$ is an uncountable set.Generally,we suppose $\pi_{1}(D)$ to be an uncountable set.

Let $x, x^{\prime} \in \pi_{1}(D)$, and $x \neq x^{\prime}$.then exists $y \in \pi_{2}(D)$, such that $(x, y),\left(x^{\prime}, y^{\prime}\right)$

$\in D$,so we have $\varepsilon_{t}(\varphi \times \varphi, \mu, v, p)=\varepsilon_{t}\left(\varphi, x, x^{\prime}, p\right)$. Hence it is easy to show that

$$
\underline{F}\left(\varphi, x, x^{\prime}, p\right)=0, \quad \bar{F}\left(\varphi, x, x^{\prime}, s\right)=1, \forall s>0 .
$$

Consequently, $\varphi$ is distributional chaotic. This completes the proof.

\section{Summary}

In this paper, we prove that $\varphi$ is Li-Yorke chaotic if and only if $\varphi \times \varphi$ is Li-Yorke chaotic; $\varphi$ is distributional chaotic if and only if $\varphi \times \varphi$ is distributional chaotic.

\section{Acknowledgements}

This work is supported by NSFC(Grant Nos.61462032), the Science and technology research project of Jiangxi Provincial Department (Grant Nos. GJJ161227), the Science and technology 
research project of Nanchang Institute of Science and Technology (Grant Nos. NGKJ-17-05, GJKJ-16-01).

\section{References}

[1] John. B. Author, and A. Friend, "Journal paper's name", Journal's name, 2001,Vol 39, No. 1, pp. 222-226.

[2] Xizhao Wang, His book's name, Publisher, Location, Year.

[3] Li. T. Y, Yorke. J. Period three implies chaos. Amer.Math. Mothly,1975, 82,pp.985--992.

[4] Berthold.S,Jaroslav.S.Measures of chaos and a spectral decomposition of dynamical systems of the interval.Trans,Amer.Math.Soc,1994,344,pp.737-754.

[5] Huang.Y, Zhou.Z.L.Two new recurrent levels for $C^{0}$-Flows.Acta.Appl.Math, 2012,118pp.125-145.

[6] Wang.L.D, Huan.S.M,Huang.G.F.A note on Schweizer-Smital chaos.Nonlinear.Anal.2008,68. 